\title{
1 Anti-allergic and anti-inflammatory activities of black 2 cumin extracts in in vitro and in vivo model systems
}

3 1

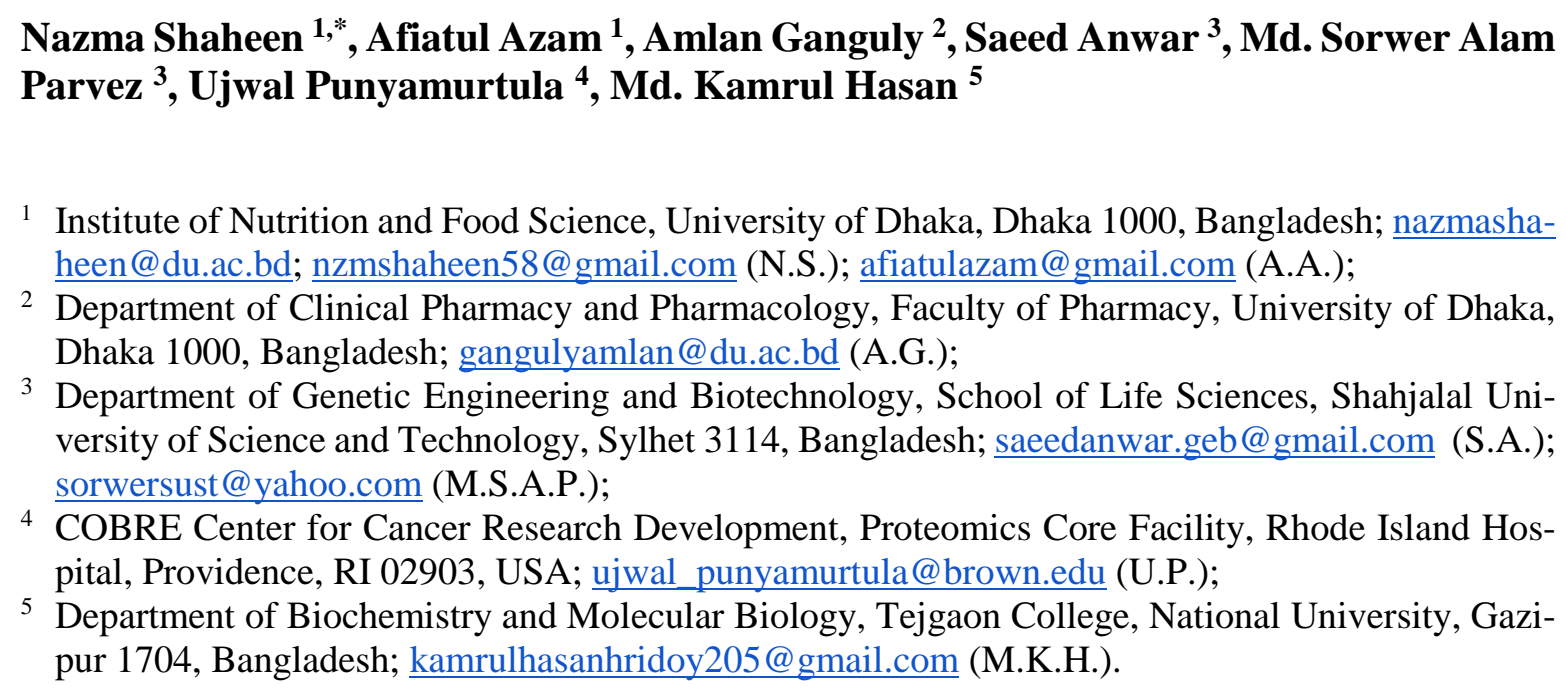

1 Institute of Nutrition and Food Science, University of Dhaka, Dhaka 1000, Bangladesh; nazmashaheen@du.ac.bd; nzmshaheen58@gmail.com (N.S.); afiatulazam@gmail.com (A.A.);

2 Department of Clinical Pharmacy and Pharmacology, Faculty of Pharmacy, University of Dhaka, Dhaka 1000, Bangladesh; gangulyamlan@du.ac.bd (A.G.);

3 Department of Genetic Engineering and Biotechnology, School of Life Sciences, Shahjalal University of Science and Technology, Sylhet 3114, Bangladesh; saeedanwar.geb@gmail.com (S.A.); sorwersust@yahoo.com (M.S.A.P.);

4 COBRE Center for Cancer Research Development, Proteomics Core Facility, Rhode Island Hospital, Providence, RI 02903, USA; ujwal_punyamurtula@brown.edu (U.P.);

5 Department of Biochemistry and Molecular Biology, Tejgaon College, National University, Gazipur 1704, Bangladesh; kamrulhasanhridoy205@gmail.com (M.K.H.).

* Correspondence:

Dr. Nazma Shaheen

Professor,

Institute of Nutrition and Food Science,

University of Dhaka,

Dhaka-1000

Contact- +8801747799562

Email-nnzmshaheen58@gmail.com, nazmashaheen@du.ac.bd

(1)

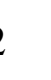




\section{Abstract}

Black cumin (Nigella sativa) is a widely used ingredient of traditional medicine for its broadspectrum pharmacological actions, including anti-allergic, bronchial asthma, and anti-inflammatory properties. We sought to evaluate BC extracts' efficacy for their anti-allergic and anti-inflammatory properties using a comprehensive in vitro, in vivo, and silico experimental setup. To investigate whether BC extract has anti-inflammatory, anti-allergic, and analgesic therapeutic potentials in vitro and in vivo. The activity of $\mathrm{BC}$ was assessed through antiallergic activity on rat basophilic leukemia-2H3 cell line, anti-inflammatory activity on J774.1A cell line, anti-inflammatory activity by carrageenan-induced rat paw edema, analgesic activity by acetic acid-induced writhing test, and ingenuity analysis of the BC extracts in inflammation control. BC exerted potent anti-allergic activity by inhibiting antigen-induced degranulation. An anti-inflammatory effect is shown by inhibiting TNF- $\alpha$ production. The acetic acid-induced writhing test shown a dose-dependent reduction of writhing number following BC administration. Rat paw edema test shown the dose-dependent reduction of paw edema volume following BC administration. Ingenuity Pathway Analysis (IPA) suggested BC extracts containing ferulic acid, p-coumaric acid, kaempferol, and quercetin can inhibit inflammation. This study suggests that bioactive compounds in BC extract act as an anti-allergic and anti-inflammatory agent by regulating several downstream and upstream

60 inflammation pathways.

61 Keywords: Black cumin; Nigella sativa; anti-allergy; anti-inflammation; edema

\section{Abbreviations}

BC, Black cumin; RBL, Rat basophilic leukemia; IPA, Ingenuity Pathway Analysis; AWA, 
66

\section{Introduction}

Inflammation is a fundamental part of the body's physiological defense mechanisms against pathogenic infections and toxic substances [1]. It is involved in the body's response to both the initial cause and the consequences of an injury. Often, however, inflammations can be triggered inappropriately, leading to tissue destruction, which in turn can result in a range of inflammatory disorders, including rheumatoid and gouty arthritis, psoriasis, and Crohn's disease [2]. Many studies suggest that a persistent inflammatory condition can be pervasive and develop into more clinically severe afflictions, including cardiovascular disease and cancer, often with fatal outcomes [3] [2] [4].

Current management strategies for inflammatory diseases include medications, relaxation, exercise, and surgery to correct joint damage. The medications used to treat inflammatory diseases, such as non-steroidal anti-inflammatory drugs, corticosteroids, cyclophosphamide, hydroxy-chloroquine, and biologic drugs, possibly minimize disease progression by lowering joint pain, swelling, and the inflammation itself [5] [6] [7] [8]. The type of management strategy is contingent on many factors, including the patient's age, medical background and comorbidity, immunity status, and the severity of the symptoms of inflammatory disease [5] [8]. However, the efficacy of these management strategies can be questionable, and even if the efficacy is satisfactory, many of these strategies are often not suitable for all patients because of associated side effects [9]. Both steroidal and non-steroidal inflammatory drugs are associated with a high range of adverse effects in the long-term [10] [11]. There is thus a constant push toward the development of an effective and curative therapeutic strategy to treat inflammatory diseases. 
Medicinal plants are an essential source of bioactive compounds with potential thera-

91 peutic efficacy [12]. Pharmacological investigations of medicinal plants can yield primers

92 for the effective treatment of inflammatory diseases [12]. Black Cumin (BC, Nigella sativa

93 L.) is a well-known medicinal plant used extensively in Unani, Ayurvedic, and Siddhi med-

94 icine for centuries [13]. This herb, endemic to South Asian and Mediterranean countries, is

95 rich in bioactive compounds, including tocopherols, alkaloids, saponins, and vitamins A and

$96 \mathrm{C}$, all of which contribute to its biological functionality [14] [15] [16]. Overwhelming evi-

dence indicates the presence of bioactive ingredients in $\mathrm{BC}$ that can counteract the underlying pathophysiology of many diseases, including cancers, inflammatory conditions, cardiovasthe probable anti-inflammatory and anti-analgesic activities of BC [19] [20]. However, only

101 a limited number of studies have explored BC's anti-inflammatory effects on subacute and chronic models of inflammation [20].

As a result, we sought to investigate the dose-response effects of the anti-inflammatory activity of $\mathrm{BC}$ in rats and mice with in vitro and in vivo anti-inflammatory, anti-allergic and anti-analgesic activities. Using these findings, the present study has taken an attempt to develop a probable mechanism of action for $N$. sativa through Ingenuity pathway analysis.

\section{Materials and Methods}

\section{Collection and preparation of black cumin samples}

Trained food sample collectors collected the samples from New market Kancha Bazar,

111 Dhaka, Bangladesh and then critical checking of BC seed samples by the expert faculty mem-

112 ber of the Department of Botanist, University of Dhaka. Preparation and processing of the 
113 collected samples were done using standard operating procedures. Processing involved dry-

114 ing of the fresh samples at $25-30^{\circ} \mathrm{C}$, grinding by an electric blender, and preserving in an

115 airtight container until analysis.

\section{Extraction procedure}

117 Multiple rounds of sequential extraction (initially by hexane/dichloromethane $(1: 1 \mathrm{v} / \mathrm{v})$

118 (Merck, Germany; hexane- 296090, dichloromethane- 270997) and then by AWA (ace-

119 tone/water/acetic acid 70:29.5:0.5) (Merck, Germany; acetone- 650501, acetic acid- A16283)

120 was performed in an accelerated solvent extraction equipment known as ASE 200 (DIONEX,

121 USA, catalogue number: 055422). A detailed description of the extraction procedure has

122 been reported elsewhere [21] [22]. While ground samples were directly mixed with Dimethyl

123 sulfoxide (DMSO) (Sigma Aldrich, \#D- 2650 Poland) to get the sample extracts for

124 assessment of anti-allergic and anti-inflammatory activities in in vitro cell line models, in

125 vivo assessments conducted in this study mixed dried AWA extracts with DMSO to prepare

126 the sample extracts.

\section{Experimental animal}

128 Swiss Albino mice (5 - 6 weeks of age; 20 - 30 grams) and Long-Evans rats (7 - 8 weeks of 129 age; 100 - 130 grams) were collected from the Animal Research Branch, International Centre

130 for Diarrheal Disease Research, Bangladesh (ICDDR, B). The animal were housed in poly-

131 vinyl cages and maintained under standard laboratory conditions (temperature $25 \pm 2{ }^{\circ} \mathrm{C}$ ) and

132 a $12 \mathrm{~h}$ light $-12 \mathrm{~h}$ dark cycles for seven days. The animals were fed on standard laboratory

133 animal diet formulated by ICDDR, B and water ad libitum. To keep the hydration rate con-

134 stant, food and water were stopped $12 \mathrm{~h}$ before the experiments. The Ethical and Animal Care

135 Committee of the Institute of Nutrition and Food Sciences, University of Dhaka, Bangladesh,

136 critically reviewed and approved this study involving in vivo models. The procedures de- 
137

138

139

scribed in this study were conducted in accordance with the Bangladesh Biosafety and Biosecurity guidelines 2019 and institutional oversight performed by qualified veterinarians $[23]$.

\section{Anti-allergic activity on rat basophilic leukemia-2H3 cell line}

Rat basophil leukemia (RBL)-2H3 cells [NIHS (JCRB), Tokyo, Japan: Cell No.: JCRB 0023] were used to study the anti-allergic activities of BC extracts. RBL-2H3 cells were grown in minimal essential medium (Eagle) containing $15 \%$ fetal calf serum. We then inoculated $05 \times 10^{5}$ cells in each well of a 24 -well plate and incubated at $37^{\circ} \mathrm{C}$ in an environment of $5 \%$ carbon dioxide for affluent growth. After overnight incubation $\left(4 \times 10^{6}\right.$ cells in each well of a 24-well plate), mouse monoclonal anti-DNP IgE (Sigma Aldrich \#A- 6661, UK) solution was added to each of the 24 wells before incubation (for two hours at $37^{\circ} \mathrm{C}$ ).

The cells were then incubated with $10 \mu \mathrm{l}$ of DNP labeled human serum albumin for 30 minutes. Supernatants were separated after lysis of cells with $500 \mu l$ of Triton $X-100$. The cell lysate $(50 \mu \mathrm{l})$ along with the collected supernatant was transferred to the 96-well plate and mixed with $100 \mu \mathrm{l}$ of $0.1 \mathrm{M}$ citrate buffer containing $3.3 \mathrm{mM}$ para-nitrophenyl-2-acetamide- $\beta$-D-glucopyranoside (Wako, Japan). The supernatant-cell lysate mix was then kept under incubation for 25 minutes at $37^{\circ} \mathrm{C}$; it was followed by an addition $(100 \mu \mathrm{l})$ of $2 \mathrm{M}$ glycine buffer of $\mathrm{pH} 10.0$ which stopped the reaction in a microplate reader and measurement of the absorbance. A stop solution was added to the lower four lanes which were used as control, and then at the same time, the substrate was added to the experimental well.

Blank without antigen and another negative control were used as a positive control with antigen and $5 \mu \mathrm{M}$ of wortmannin (Wako, Japan) solution. Blank, negative control, and positive control were prepared by adding $490 \mu 1$ of modified Tyrode's buffer and 200 folds diluted wortmannin solution. 
Anti-inflammatory potential using J774.1A cell assay was assessed using the method as

163 reported by Herath et al. [24]. Shortly, ground BC seed samples were mixed with DMSO to

164 prepare $4 \mathrm{mg} / \mathrm{ml}$ concentration and final strength of $40 \mu \mathrm{g} / \mathrm{ml}$ was employed in the assay me-

165 dia. For the assay, a clear supernatant was obtained by centrifuging that mixture at $3000 \mathrm{rpm}$

166 for 5 minutes. Mouse macrophages J774A.1 cell line [NIHS (JCRB) 9108] were cultured in

167 Dulbecco's Modified Eagle Media (DMEM) (Sigma Aldrich \#D-5030, USA) with 10\% fetal

168 calf serum and penicillin and streptomycin (Sigma Aldrich \#F-7524, UK) at a concentration

169 of $100 \mathrm{U} / \mathrm{mL}$. The cells were incubated at $37^{\circ} \mathrm{C}$ in the incubator with an atmosphere of $5 \%$

$170 \mathrm{CO} 2,95 \%$ air.

The cell suspension with the concentration of $5.0 \times 10^{5}$ cell $/ \mathrm{ml}(200 \mu 1 /$ well $)$ was placed in 96 well culture plates and incubated overnight $\left(37^{\circ} \mathrm{C}, 5 \% \mathrm{CO}-95 \%\right.$ air $)$. Overnight cultured cells washed thrice with Hank's solution (Sigma Aldrich \#6648, UK) $\left(37^{\circ} \mathrm{C}\right)$ after removal of culture media prior to the addition of $180 \mu 1$ DMEM and followed $20 \mu 1$ of 10 times diluted sample extracts (final concentration, $40 \mu \mathrm{g} / \mathrm{ml}$ ) to the each well without lipopolysaccharides (LPS). On the other hand, $160 \mu \mathrm{l}$ DMEM with $20 \mu \mathrm{l}$ of LPS (final concentration, $1.0 \mu \mathrm{g} / \mathrm{ml}$ ) after the addition of the test samples and incubated further for more than four

179 hours. Cell supernatant was then collected for the assay of the TNF- $\alpha$ production, assayed by using Mouse TNF- $\alpha$ ELISA kit (Ready-Set-Go, eBioscience, USA). In brief, $50 \mu 1$ of TNF$\alpha$ capture antibody (250 times diluted) was added into 96 well plates and kept in the refrigerator $\left(4^{\circ} \mathrm{C}\right)$ overnight. The plate was washed with $300 \mu \mathrm{l}$ of PBS-T (0.05\% Tween20 in PBS)

1833 times in a sera washer (Model MW-96F., Biotech, Japan) and $100 \mu 1$ of assay diluents 184 solution was added as blocking solution. After incubation for 1 hour at room temperature, the plate was washed and $50 \mu 1$ of cell supernatant /standard. TNF- $\alpha$ was added into the wells according to plate design. Sample extract-treated cell supernatant was diluted 10 times with 
$1000 \mathrm{pg} / \mathrm{ml}$ and further incubated for 2 hour at RT. After at least $2 \mathrm{hrs}$ incubation, plates were

189 washed again and TNF- $\alpha$ detection antibody (250 times diluted) was added (50 $\mu 1)$ into each

190 well and further incubated for 1hour. Avidin- HRP antibody solution (250 times diluted) was

191 added in the same volume as earlier after washing and kept 30 minutes at RT. Finally, $50 \mu 1$

192 of TMB-HRP substrate solution was added and incubated for $15 \mathrm{~min}$., a stop solution $(25 \mu \mathrm{l})$

193 was added into each well to stop the reaction and the absorbance was read in the microplate

194 reader (model 550; Bio Rad, CA, USA) at $450 \mathrm{~nm}$. From the absorbance data, the inhibition

195 effect of food extracts on TNF- $\alpha$ production was calculated from the standard graph. Dose-

196 response was done for the samples which had an inhibitory effect on TNF- $\alpha$ production at

197 the concentrations of $40,10,3$, and $1 \mu \mathrm{g} / \mathrm{ml}$.

\section{Assessment of anti-inflammatory activity by carrageenan-induced rat paw edema}

Evaluation of anti-inflammatory activity was conducted in vivo in the Long Evans rat model. At first $200 \mathrm{mg}$ of samples (i.e., dried AWA extract) were mixed with $5.0 \mathrm{ml}$ of DMSO in a shaking-incubator at $130 \mathrm{rpm}$ overnight. Then the mixture was centrifuged at $3000 \mathrm{rpm}$ for 5.0 minutes. Then the supernatant was collected and pipetted $500 \mu \mathrm{L}$ of aliquots to store at $25^{\circ} \mathrm{C}$.

To determine anti-inflammatory activity, edema was induced by the phlogistic agent carrageenan [25]. The control group $(\mathrm{n}=3)$ received normal saline per os (p.o) $(10 \mathrm{ml} / \mathrm{kg})$, while group 2 received a standard drug, Diclofenac sodium (50 mg/kg, p.o). The rest of the groups were given $800(n=3), 400(n=3)$, and $200 \mathrm{mg} / \mathrm{kg}$ body weight $(\mathrm{n}=3)$ p.o. of extract preparations. Thirty minutes after administering sample extracts, each rat received $0.1 \mathrm{ml}$ of $1 \%(\mathrm{w} / \mathrm{v})$ carrageenan (injected into the sub-plantar region of the right hind paw subcutaneously) (Sigma Aldrich, \# C-1013, Germany). Paw volumes of the right hind paw of each rat were measured by plethysmometer before and 1,2,3, and 4 hours after carrageenan injection to determine the edema volume. The hind paw volume was evaluated for anti-inflammatory 
213 activity and expressed as \% inhibition of the hind paw volume, which was calculated by the

214 following equation:

$215 \%$ inhibition $=[(\mathrm{Vc}-\mathrm{Vt}) / \mathrm{Vc}] \times 100$

216 Here, $\mathrm{Vc}=$ average paw volume of the control group, and $\mathrm{Vt}=$ Average paw volume of

217 the treated group.

\section{Assessment of analgesic activity by acetic acid-induced writhing test}

Peripheral analgesic activity of the extracts was determined by the acetic-acid-induced writhing inhibition method in mice [26]. The control group (n=3) (group 1) received normal intraperitoneally with $0.6 \%$ acetic $(10 \mathrm{ml} / \mathrm{kg})$. Later, the writhing numbers of each mouse were observed for 10 minutes. To evaluate the level of analgesia, writhing numbers of the sample treated mice were compared with the writhing numbers of the control groups. Percent-inhibition of writhing was calculated using the following equation:

Here, $\mathrm{Wc}=$ Average number of the writhing of the control group, and $\mathrm{Wt}=$ Average number of the writhing of the treated group.

\section{Ingenuity analysis of the BC extracts in inflammation control}

We evaluated the effects of the compounds extracted from BC using the knowledgeplored new ingenuity-based pathways that showed the relationship between the genes involved in the biosynthetic pathways of inflammation-related compounds (e.g., arachidonic

236 acids, prostaglandins, and leukotrienes) and compounds in the BC extracts, which includes

237 ferulic acid, quercetin, p-coumaric acid, and kaempferol. The shortest possible pathways 
238 were generated individually for each compound where the compounds in the BC extracts

239 were set as the initial compound, and inflammation-related compounds were selected as the 240 target.

\section{$241 \quad$ Results}

242 In vitro screening for anti-allergic effect

As compared to the negative control, $\mathrm{BC}$ extracts produced $46.9 \%(\mathrm{SD} \pm 7.1)$ inhibition

244 of the antigen-induced degranulation of RBL-2H3 cells. AWA extracts produced increased

245 inhibition compared to the DMSO extracts for 5, 10, 20, and $40 \mu \mathrm{g} / \mathrm{ml}$ concentration. How246 ever, at higher concentrations, e.g., $40 \mu \mathrm{g} / \mathrm{ml}$, both extracts produced comparable inhibition 247 (Figure 1).

248 In vitro screening for anti-inflammatory effect

249 In the preliminary evaluation of anti-inflammatory effects on J774A.1 cell, $40 \mu \mathrm{g} / \mathrm{mL}$ of 250 DMSO extracts of BC resulted in significant (70\%) inhibition of TNF- $\alpha$ production. Dose251 response analysis showed that $1,3,10$, and $40 \mu \mathrm{g} / \mathrm{mL}$ of DMSO extracts could produce up 252 to $70 \%$ inhibition in the TNF- $\alpha$ production.

\section{In vivo anti-inflammatory activity}

Four hours after carrageenan injection, the maximum volume of edema in control Long

255 Evans adult rat models was $1.18 \pm 0.03 \mathrm{ml}$. Another group of rats pretreated with Diclofenac

256 sodium (reference drug; $50 \mathrm{mg} / \mathrm{kg}$, p.o), showed significantly reduced $(\mathrm{p}<0.05)$ edema in 257 the paw. Dose-response analysis of DMSO extract of BC produced a significant inhibition 258 of paw edema in Long Evans adult rats in a dose-dependent manner at 200, 400, and 800 $259 \mathrm{mg} / \mathrm{kg}$, p.o administration of carrageenan $(\mathrm{p}<0.05)($ Table 1$)$. 
The maximum inhibition of rat paw edema by BC was noted 4 hours after administration

261 of carrageenan at a dose of $800 \mathrm{mg} / \mathrm{kg}$, p.o. compared to the control but less than the reference

262 drug. Figure 2 shows the changes of percent inhibition of carrageenan-induced paw edema

263 volume at $1,2,3$, and $4 \mathrm{~h}$ at the doses of $800 \mathrm{mg} / \mathrm{kg}$, p.o by the DMSO extract of BC when

264 compared to the control.

\section{In vivo analgesic activity}

The effects of DMSO extract of BC on $0.6 \%$ acetic acid-induced writhing in Swiss albino

mice are summarized in Table 2. A dose-dependent and significant $(\mathrm{p}<0.05)$ reduction in the

number of abdominal constrictions induced by intraperitoneal administration of $0.6 \%$ acetic to that of control.

The maximum inhibition of rat paw edema by $\mathrm{BC}$ was noted 4 hours after admin-

274 istration of carrageenan at a dose of $800 \mathrm{mg} / \mathrm{kg}$, p.o. compared to the control but less than

275 the reference drug. Figure 2 shows the changes of percent decrease in carrageenan-induced

276 paw edema volume at first, second, third, and fourth hours post-administration of 800

$277 \mathrm{mg} / \mathrm{kg}$, p.o of DMSO extract of BC when compared to the control.

In silico analysis provides insights into the mechanism of how $\mathrm{BC}$ extracts reduce in-

\section{flammation}

The in-silico analysis of the bioactive compounds of BC illustrates a facet reactome de-

281 picting potential models of how BC extracts reduce inflammation in humans (Figure 3, S1

282 Table). Both prostaglandin and leukotriene synthesize from the arachidonic acid. Quercetin,

283 p-coumaric acid, and kaempferol reduce the expression of myc, MAPK, EGFR, and TNF, all 
of which are involved in the upregulation of the expression of arachidonic acid. Conse-

285 quently, quercetin, p-coumaric acid, and kaempferol downregulate the expression of arachidonic acids.

Ferulic acid, another component in the BC extract, negatively induces the synthesis of phospholipids. Phospholipid biosynthesis is critically important for the synthesis of arachidonic acids. Also, ferulic acids upregulate the expression of HMOX and nrf2, resulting in the reduction of prostaglandin E2 (PGE2). Moreover, all the compounds of the Nigella extract

291 directly reduce the expression of $\operatorname{cox} 1$ and $\operatorname{cox} 2$, two essential factors for the synthesis of PGE2. Collectively, ferulic acid, quercetin, p-coumaric acid, and kaempferol help reduce inflammation by interfering with the extended arachidonic acid pathway (Figure 3; S1 Table).

\section{Discussion}

In this study, we demonstrate the in vitro dose-response of the DMSO extract of $\mathrm{BC}$ to evaluate the anti-inflammatory and analgesic activities in the experimental models. The inhibition of the antigen-induced degranulation at the same concentration of 41 food samples was assayed in the rat basophilic leukemia RBL-2H3 cell line (data not shown). A further dose-response $(40,20,10$ and $5 \mu \mathrm{g} / \mathrm{mL})$ assay was carried out to confirm their anti-allergic activity of the bioactive compound as present in food samples and only DMSO, as well as AWA extract of $\mathrm{BC}$, exhibited dose-response which support the concept that anti-allergic activity belongs to the phenolic compounds. Recent research findings showed that polyphe-

304 nols, widely distributed among fruits, vegetables, and herbs, are widespread for their antioxidant capacity [29]. They also reported that some of them could exert anti-allergic activities [30] [31] [29]. 
formation of edema occurs in a biphasic process. Mast cells around the damaged tissue re-

310 leased prostaglandin with histamine and serotonin mediate the first phase $(1-2 \mathrm{~h})$ of the

311 carrageenan model. Bradykinin, leukotrienes, polymorphonuclear cells, and continuous se-

312 cretion of prostaglandins from macrophages tissue mediate the second phage $(3-5 h)$ of the

313 carrageenan model [32] [25]. Our study indicates that the DMSO extracts of BC exhibited

314 significant $(\mathrm{p}<0.05)$ inhibition of paw edema in rats at the doses of 200,400 , and $800 \mathrm{mg} / \mathrm{kg}$,

315 p.o in the second phases of inflammatory response. DMSO extracts of BC at the dose of 800

$316 \mathrm{mg} / \mathrm{kg}$ produced the maximum inhibition of carrageenan-induced paw edema volume. The

317 result of the present study showed the maximum inhibition of carrageenan-induced paw

318 edema volume in rats by $\mathrm{BC}$ in the second phase of post carrageenan injection at 3-5 h, maybe

319 due to the modulatory principles acting with the prostaglandin alley. Moreover, the present

320 study supports the previous findings that BC seed polyphenol plays a crucial role as protec-

321 tive factors against

"Acetic acid-induced writhing experiment" is a well-known protocol for assessing the analgesic potency of medicinal products [33]. Intraperitoneal injection of acetic acid that triggers pain sensation is due to the prostaglandins and lipoxygenase products from arachidonic acid liberated from phospholipids by cyclooxygenase [34]. Thus, the significant

$326(\mathrm{p}<0.05)$ reduction of writhing in this study by DMSO extracts of BC at the doses of 200, 327400 , and $800 \mathrm{mg} / \mathrm{kg}$, p.o suggest analgesic activity peripherally mediated through inhibition 328 of prostaglandins and other endogenous pain mediators. The highest concentration (800 $329 \mathrm{mg} / \mathrm{kg}$ ) of the test samples exhibited a peak of analgesic effect significantly $(\mathrm{p}<0.05)$ in the 330 acetic acid-induced writhing test. These results indicate the peripheral analgesic potential of 331 the DMSO extracts of the test samples which could be exhibited due to the suppression of 332 peritoneal surface receptors through inhibited cyclooxygenase activity. The current study 333 findings show concordance with previous findings that BC seed polyphenol inhibits acetic 334 acid-induced writhing in the mouse model [35]. 
Through an examination of the literature, we also note how to present findings confirm

336 previous work in the field. Specifically, the in vitro and in vivo experiments performed in this

337 study are in good agreement with those of prior experiments on BC's anti-inflammatory ac-

338 tivity, lending credence to the idea that $\mathrm{BC}$ is a potent medicinal plant for therapeutic uses.

339 In addition to the wet-lab analysis, ingenuity pathway analysis provides evidence for the

340 role of the Nigella extracts in the reduction of inflammation. Earlier studies revealed that

341 some molecules including myc, MAPK, EGFR, TNF, are involved in the cellular responses

342 for inducing the inflammation [36] [37] [38] [39]. We have found that compounds in the

343 Nigella extracts, including ferulic acid, quercetin, p-coumaric acid, and kaempferol, down-

344 regulates the inflammation-inducing signaling molecules in humans (Figure 3). The potential

345 role of the activation of $\mathrm{pKA}, \mathrm{HMOX}$, and nrf2 pathways led to anti-inflammatory effects

346 has been reported in many research groups [40] [41] [42] [43]. Our analysis uncovered that

347 compounds in the Nigella extracts upregulate the pKA, HMOX, and nrf2 (Figure 3).

Furthermore, we have shown the potential aspects of the anti-inflammatory mecha-

349 nism of BC that were previously only speculated upon. For example, Nasuti et al. found BC

350 to reduce only the acute inflammation suggested that BC's mechanism of action is tied to

351 interleukins, TNF, and prostaglandins [44]. In the ingenuity pathway analysis, we have

352 shown various components of BC act through IL32, IL8, IL1, TNF, and PGE2 to reduce

353 inflammation. In another study, researchers discovered that BC could decrease either IL-6

354 levels of IL-1B levels, depending on its storage conditions [45]. These findings establish a

355 connection between BC and interleukins in BC's anti-inflammatory activity, which we have

356 at least partially outlined in Figure 3. Similarly, other researchers speculated that BC WSE

357 inhibits $\mathrm{COX} 2$ and that $\mathrm{BC}$ inhibits prostaglandin synthesis through interaction with $\mathrm{COX}-1$

358 and COX-2 [46]. Both hypotheses are supported by the results of our Ingenuity pathway

359 analysis, as depicted in Figure 3. Furthermore, Babar et al. suggested that phenols/polyphe-

360 nols in BC might be responsible for its anti-inflammatory activity [46]. Through our pathway 
analysis, we have found that these phenols and polyphenols are ferulic acid, p-coumaric acid,

362 kaempferol, and quercetin. It is speculated that the inhibitory effect on the secretion of leu-

363 kotrienes and prostaglandins by thymoquinone may be responsible for BC's anti-inflamma-

364 tory activity [47]. We have shown that this is the case, although, instead of thymoquinone,

365 quercetin and ferulic acid were found to be the components of $\mathrm{BC}$ responsible for this aspect

366 of its anti-inflammatory mechanism. In another study, N. sativa showed superior activity in

367 a screening of Nigella species for in vitro inhibition of PGE2 production catalyzed by COX1

368 and COX2 [48]. This lends credence to the mechanistic schematic we have depicted in Figure

3693 ; as is shown in that diagram, several components of BC act to inhibit COX1/COX2 activity,

370 which is indeed necessary to produce PGE2. In yet another study, researchers discovered that

371 levels of erk are decreased upon $N$. sativa treatment of damaged rat lungs [49]. The erk re-

372 pression discussed here is supported by our mechanistic analysis, which has found that

373 kaempferol, a component of $N$. sativa, inhibits ERK1/2 activity. As evidenced by these ex-

374 amples from the literature, the results we have found build on previous research to provide

375 crucial insight into the medicinal properties of a common natural product.

376 Together with the in vitro and in vivo studies, the in silico analysis in this study provides

377 strong evidence that the bioactive compounds in BC extract act as an anti-inflammatory agent

378 by regulating several downstream and upstream pathways of inflammation.

\section{Conclusion}

380 In summary, the findings of the present study support that a BC extract has anti-inflamma-

381 tory, anti-allergic, and analgesic activity. This study, involving in vitro and in vivo systems,

382 indicates the possibility that $\mathrm{BC}$ extract might help treat pathologies that involve chronic

383 inflammation and pain. Also, in silico analyses suggested that the phenolic compounds in BC

384 extracts may play a key role in inhibiting inflammatory reactions in human? Besides, through 
the Ingenuity pathway analysis, the present study has supported and elaborated on sugges-

386 tions found in the literature regarding BC's biochemical mechanism of action. Thus, in un-

387 derstanding how to present findings relate to prior work in the field, we have gained insight

388 into the vitality of this research for future efforts to study the anti-inflammatory effects of $N$.

389 sativa. Future studies should focus on elucidating the mechanistic pathways through which

390 compounds in BC extracts exert such pharmacologic effects to prevent inflammation.

391 Supplementary Materials: Table S1: Components of the reactome Nigella sativa works on 392 to impact on human inflammations.

393 Acknowledgments: We thank Aftab Uz Zaman Noor, MSc (Université Claude Bernard

394 Lyon 1, France) for his in-kind assistance for conducting the silico study.

395 Author Contributions: Conceptualization, N.S. M.K.H., and S.A.; methodology, N.S., 396 A.A., A.G., S.A., M.K.H. and M.S.A.P.; software, S.A., and M.S.A.P; validation and scruti397 nization, N.S., M.K.H., and U.P; investigation, N.S., A.A., A.G., S.A. and M.S.A.P.; formal 398 analysis, N.S., A.A., A.G., S.A., and M.S.A.P.; writing-original draft preparation, A.A., 399 A.G., and S.A.; writing — review and editing, N.S., M.K.H., and U.P.; supervision, N.S.; pro400 ject administration, N.S., and M.K.H.; funding acquisition, N.S.

401 Competing Interest: The authors do not have any conflicting interest.

402 Approval for animal experiments: Animals were housed at the Animal Housing Facility 403 of Institute of Nutrition and Food Sciences, University of Dhaka, Bangladesh, following 404 guidelines by their Ethics Committee for the Treatment of Laboratory Small Animals. All 405 procedures were reviewed and approved by the Ethical and Animal Care Committee of the 406 Biological Science faculty, University of Dhaka, Bangladesh. The committee's reference 407 number is not available.

Approval for human experiments: Not applicable.

409 Approval for experimental procedures: All experimental procedures were approved by the 410 internal committee of Biological Science faculty headed by Dean of Bioscience faculty, Uni411 versity of Dhaka, Bangladesh. 
Informed consent: Informed consent was obtained from all individual participants included

414 in the study.

415 Data availability: Authors always welcome to share the data required for reviewers and

other researchers.

Funding: The author(s) received no specific funding for this project.

\section{References}

[1] R. Medzhitov, "Origin and physiological roles of inflammation," Nature, vol. 454, no. 7203. Nature Publishing Group, pp. 428-435, Jul. 24, 2008, doi: 10.1038/nature07201.

[2] D. Okin and R. Medzhitov, "Evolution of inflammatory diseases," Current Biology, vol. 22, no. 17. Curr Biol, Sep. 11, 2012, doi: 10.1016/j.cub.2012.07.029.

[3] D. Laveti et al., "Anti-inflammatory treatments for chronic diseases: A review,"

[5] H. Cheng, "Genetic Variation in Resistance to Inflammation and Infectious

[4] I. Roifman, P. L. Beck, T. J. Anderson, M. J. Eisenberg, and J. Genest, "Chronic inflammatory diseases and cardiovascular risk: A systematic review," Canadian Journal of Cardiology, vol. 27, no. 2. Pulsus Group Inc., pp. 174-182, 2011, doi: 10.1016/j.cjca.2010.12.040. Inflammation and Allergy - Drug Targets, vol. 12, no. 5. Inflamm Allergy Drug Targets, pp. 349-361, 2013, doi: 10.2174/18715281113129990053.

[6] B. R. R. De Mattos et al., "Inflammatory bowel disease: An overview of immune mechanisms and biological treatments," Mediators of Inflammation, vol. 2015. Hindawi Publishing Corporation, 2015, doi: 10.1155/2015/493012.

[7] A. Diaz-Borjon, C. M. Weyand, and J. J. Goronzy, “Treatment of chronic inflammatory diseases with biologic agents: Opportunities and risks for the elderly," Experimental Gerontology, vol. 41, no. 12. Exp Gerontol, pp. 1250-1255, Dec. 2006, doi: 10.1016/j.exger.2006.10.010.

[8] M. S. (Editor) Jean-Marc Cavaillon (Editor), "Inflammation: From Molecular and Cellular Mechanisms to the Clinic, 4 Volume Set | Wiley." https://www.wiley.com/enus/Inflammation\%3A+From+Molecular+and+Cellular+Mechanisms+to+the+Clinic \%2C+4+Volume+Set-p-9783527338993 (accessed Dec. 09, 2020).

[9] P. Li, Y. Zheng, and X. Chen, "Drugs for autoimmune inflammatory diseases: From small molecule compounds to anti-TNF biologics," Frontiers in Pharmacology, vol. 8, no. JUL. Frontiers Media S.A., Jul. 12, 2017, doi: 10.3389/fphar.2017.00460. 
[10] A. P. Gor and M. Saksena, "Adverse drug reactions of nonsteroidal antiinflammatory drugs in orthopedic patients," J. Pharmacol. Pharmacother., vol. 2, no. 1, pp. 26-29, Jan. 2011, doi: 10.4103/0976-500X.77104.

[11] N. A. Hanania, K. R. Chapman, and S. Kesten, "Adverse effects of inhaled corticosteroids," The American Journal of Medicine, vol. 98, no. 2. Am J Med, pp. 196-208, 1995, doi: 10.1016/S0002-9343(99)80404-5.

[12] A. G. Atanasov et al., "Discovery and resupply of pharmacologically active plantderived natural products: A review," Biotechnology Advances, vol. 33, no. 8. Elsevier Inc., pp. 1582-1614, Dec. 01, 2015, doi: 10.1016/j.biotechadv.2015.08.001.

[13] P. J. Houghton, R. Zarka, B. De Las Heras, and J. R. S. Hoult, "Fixed oil of Nigella sativa and derived thymoquinone inhibit eicosanoid generation in leukocytes and membrane lipid peroxidation," Planta Med., vol. 61, no. 1, pp. 33-36, 1995, doi: $10.1055 / \mathrm{s}-2006-957994$.

[14] M. S. Butt and M. T. Sultan, "Nigella sativa: Reduces the risk of various maladies," Crit. Rev. Food Sci. Nutr., vol. 50, no. 7, pp. 654-665, 2010, doi: 10.1080/10408390902768797.

[15] S. S. A. A. H. M. S. U. A. A. R. M. S. Javed, "Nutritional, phytochemical potential and pharmacological evaluation of Nigella Sativa (Kalonji) and Trachyspermum Ammi (Ajwain)," J. Med. Plants Res., vol. 6, no. 5, pp. 768-775, Feb. 2012, doi: 10.5897/jmpr11.1341.

[16] P. Paarakh, "Nigella sativa Linn.- A comprehensive review | Request PDF." https://www.researchgate.net/publication/282051619_Nigella_sativa_Linn_A_comprehensive_review (accessed Dec. 09, 2020).

[17] E. Z.; S. T. G. D. N. E. Dajani, "Overview of the preclinical pharmacological properties of Nigella sativa (black seeds): a complementary drug with historical and clinical significance - PubMed," Accessed: Dec. 09, 2020. https://pubmed.ncbi.nlm.nih.gov/28195061/.

[18] A. Tavakkoli, V. Mahdian, B. M. Razavi, and H. Hosseinzadeh, "Review on clinical trials of black seed (Nigella sativa ) and its active constituent, thymoquinone," Journal of Pharmacopuncture, vol. 20, no. 3. Korean Pharmacopuncture Institute, pp. 179-193, Sep. 01, 2017, doi: 10.3831/KPI.2017.20.021.

[19] M. Ikhsan, N. Hiedayati, K. Maeyama, and F. Nurwidya, "Nigella sativa as an antiinflammatory agent in asthma," BMC Res. Notes, vol. 11, no. 1, p. 744, Oct. 2018, doi: 10.1186/s13104-018-3858-8.

[20] H. N. Pise and S. L. Padwal, "Evaluation of anti-inflammatory activity of nigella sativa: An experimental study," Natl. J. Physiol. Pharm. Pharmacol., vol. 7, no. 7, pp. 707-711, 2017, doi: 10.5455/njppp.2017.7.0204705032017.

[21] "Hydrophilic antioxidant capacities and total phenol content of seasonal fruits of Bangladesh - PubMed," Malays. J. Nutr., vol. 18, no. 3, pp. 355-362, Dec. 2012, https://pubmed.ncbi.nlm.nih.gov/24568076/. 
"Evaluation of functional potentiality of selected commonly consumed foods of Bangladesh," Funct. Foods Heal. Dis., vol. 6, no. 11, p. 735, Nov. 2016, doi: 10.31989/ffhd.v6i11.278.

[23] "Bangladesh Biosafety and Biosecurity Guidelines for Handling and Disposal of Biohazardous Materials First Edition Bangladesh Biosafety and Biosecurity Society,” 2019. Accessed: May 31, 2021. https://bdbiosafetysecurity.org/uploads/documents/Bangladesh_Biosafety_and_Bios ecurity_Guidelines.pdf?fbclid=IwAR1xpI5NX5I4gt68zjKwMJ_eUGFzbfk4PdZ4ZC6_i7-dR00k2xTyu6E4o8.

[24] H. M. T. Herath, Y. Takano-Ishikawa, and K. Yamaki, "Inhibitory Effect of Some Flavonoids on Tumor Necrosis Factor- $\alpha$ Production in LipopolysaccharideStimulated Mouse Macrophage Cell Line J774.1," J. Med. Food, vol. 6, no. 4, pp. 365-370, 2003, doi: 10.1089/109662003772519930.

[25] C. A. Winter, E. A. Risley, and G. W. Nuss, "Carrageenin-Induced Edema in Hind Paw of the Rat as an Assay for Antiinflammatory Drugs," Proc. Soc. Exp. Biol. Med., vol. 111, no. 3, pp. 544-547, 1962, doi: 10.3181/00379727-111-27849.

[26] B. A. Whittle, "The use of changes in capillary permeability in mice to distinguish between narcotic and nonnarcotic analgesics," Br. J. Pharmacol. Chemother., vol. 22, no. 2, pp. 246-253, 1964, doi: 10.1111/j.1476-5381.1964.tb02030.x.

[27] L. Chindelevitch et al., "Causal reasoning on biological networks: Interpreting transcriptional changes," Bioinformatics, vol. 28, no. 8, pp. 1114-1121, Apr. 2012, doi: 10.1093/bioinformatics/bts090.

[28] A. Krämer, J. Green, J. Pollard, and S. Tugendreich, "Causal analysis approaches in ingenuity pathway analysis," Bioinformatics, vol. 30, no. 4, pp. 523-530, Feb. 2014, doi: 10.1093/bioinformatics/btt703.

[29] D. P. Xu et al., "Natural antioxidants in foods and medicinal plants: Extraction, assessment and resources," International Journal of Molecular Sciences, vol. 18, no. 1. MDPI AG, Jan. 05, 2017, doi: 10.3390/ijms18010096.

[30] T. Juríková, "Polyphenols and their Mechanism of Action in Allergic Immune ResponseImmune Response," Glob. J. Allergy, vol. 1, no. 2, pp. 037-039, Oct. 2015, doi: $10.17352 / 2455-8141.000008$.

[31] A. Singh, S. Holvoet, and A. Mercenier, "Dietary polyphenols in the prevention and treatment of allergic diseases," Clinical and Experimental Allergy, vol. 41, no. 10. Clin Exp Allergy, pp. 1346-1359, Oct. 2011, doi: 10.1111/j.13652222.2011.03773.x.

[32] M. Di Rosa, J. P. Giroud, and D. A. Willoughby, "Studies of the mediators of the acute inflammatory response induced in rats in different sites by carrageenan and turpentine," J. Pathol., vol. 104, no. 1, pp. 15-29, 1971, doi: 10.1002/path.1711040103.

[33] M. Afzal et al., "Anti-inflammatory and analgesic potential of a novel steroidal derivative from Bryophyllum pinnatum," Fitoterapia, vol. 83, no. 5, pp. 853-858, 
Jul. 2012, doi: 10.1016/j.fitote.2012.03.013.

[34] R. Fish, P. Danneman, M. Brown, and A. Karas, Anesthesia and Analgesia in Laboratory Animals. Elsevier Inc., 2008.

[35] A. Ghannadi, V. Hajhashemi, and H. Jafarabadi, "An investigation of the analgesic and anti-inflammatory effects of Nigella sativa seed polyphenols," J. Med. Food, vol. 8, no. 4, pp. 488-493, Dec. 2005, doi: 10.1089/jmf.2005.8.488.

[36] J. R. Bradley, “TNF-mediated inflammatory disease," Journal of Pathology, vol. 214, no. 2. J Pathol, pp. 149-160, Jan. 2008, doi: 10.1002/path.2287.

[37] B. Kaminska, "MAPK signalling pathways as molecular targets for antiinflammatory therapy - From molecular mechanisms to therapeutic benefits," in Biochimica et Biophysica Acta - Proteins and Proteomics, Dec. 2005, vol. 1754, no. 1-2, pp. 253-262, doi: 10.1016/j.bbapap.2005.08.017.

[38] S. Rayego-Mateos et al., "Role of epidermal growth factor receptor (EGFR) and its ligands in kidney inflammation and damage," Mediators of Inflammation, vol. 2018. Hindawi Limited, 2018, doi: 10.1155/2018/8739473.

[39] F. Sipos, G. Firneisz, and G. Muzes, "Therapeutic aspects of c-MYC signaling in inflammatory and cancerous colonic diseases," World Journal of Gastroenterology, vol. 22, no. 35. Baishideng Publishing Group Co., Limited, pp. 7938-7950, Sep. 21, 2016, doi: 10.3748/wjg.v22.i35.7938.

[40] M. Alcaraz, P. Fernandez, and M. Guillen, "Anti-Inflammatory Actions of the Heme Oxygenase-1 Pathway," Curr. Pharm. Des., vol. 9, no. 30, pp. 2541-2551, Mar. 2005, doi: 10.2174/1381612033453749.

[41] G. M. Campo et al., "The inhibition of hyaluronan degradation reduced proinflammatory cytokines in mouse synovial fibroblasts subjected to collagen-induced arthritis," J. Cell. Biochem., vol. 113, no. 6, pp. 1852-1867, Jun. 2012, doi: $10.1002 / \mathrm{jcb} .24054$.

[42] G. M. Campo et al., "Protein kinase a mediated anti-inflammatory effects exerted by adenosine treatment in mouse chondrocytes stimulated with IL-1 $\beta$," BioFactors, vol. 38, no. 6, pp. 429-439, Nov. 2012, doi: 10.1002/biof.1040.

[43] W. Tu, H. Wang, S. Li, Q. Liu, and H. Sha, "The Anti-Inflammatory and AntiOxidant Mechanisms of the Keap1/Nrf2/ARE Signaling Pathway in Chronic Diseases," Aging Dis., vol. 10, no. 3, p. 637, 2019, doi: 10.14336/ad.2018.0513.

[44] N. Cinzia et al., "Effects of early life permethrin exposure on spatial working memory and on monoamine levels in different brain areas of pre-senescent rats," Toxicology, vol. 303, pp. 162-168, Jan. 2013, doi: 10.1016/j.tox.2012.09.016.

[45] L. Bordoni et al., "Antioxidant and anti-inflammatory properties of nigella sativa oil in human pre-adipocytes," Antioxidants, vol. 8, no. 2, Feb. 2019, doi: 10.3390/antiox8020051.

[46] Z. M. Babar et al., "In vivo anxiolytic and in vitro anti-inflammatory activities of water-soluble extract (WSE) of Nigella sativa (L.) seeds," Nat. Prod. Res., 2019, doi: 
[47] M. U. Bashir, H. J. Qureshi, and T. Saleem, "Comparison of anti-inflamatory activity of Nigella Sativa and Diclofenac Sodium in Albino rats," J. Ayub Med. Coll. Abbottabad, vol. 27, no. 3, pp. 523-526, 2015, Accessed: Dec. 09, 2020. https://pubmed.ncbi.nlm.nih.gov/26720998/.

[48] P. Landa, P. Marsik, J. Havlik, P. Kloucek, T. Vanek, and L. Kokoska, "Evaluation of antimicrobial and anti-inflammatory activities of seed extracts from six Nigella species," J. Med. Food, vol. 12, no. 2, pp. 408-415, Apr. 2009, doi: 10.1089/jmf.2007.0600.

[49] A. Aslan, D. Boydak, M. I. Can, and T. Kuloglu, "Nigella sativa improves the carbon tetrachloride-induced lung damage in rats through repression of erk/akt pathway," Bangladesh J. Pharmacol., vol. 10, no. 3, pp. 654-659, Jul. 2015, doi: 


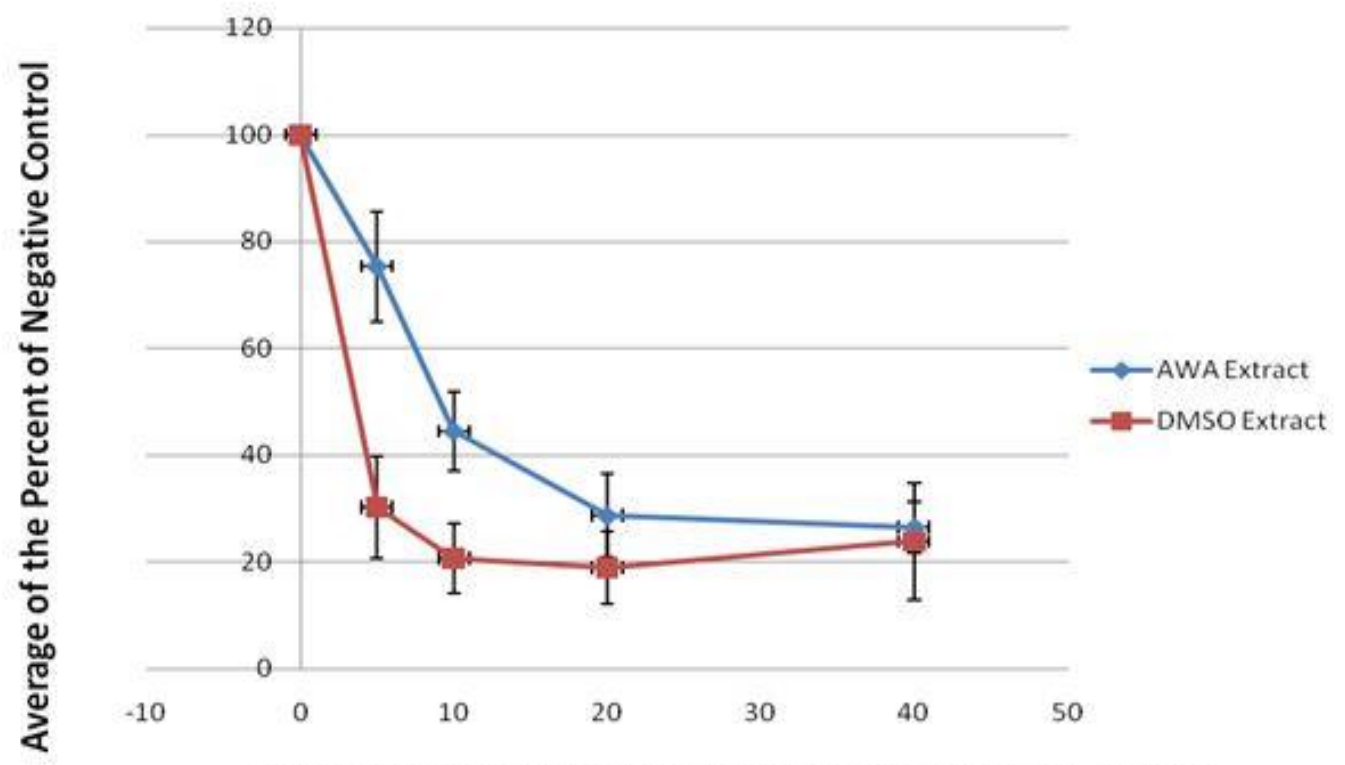

Fig.1. Comparison of dose-response of the antigen induced degranulation of BC extracts by DMSO (red color) and AWA (blue color) as percentages of negative control at the level of 5,10,20 and $40 \mu \mathrm{g} / \mathrm{ml}$ concentration.

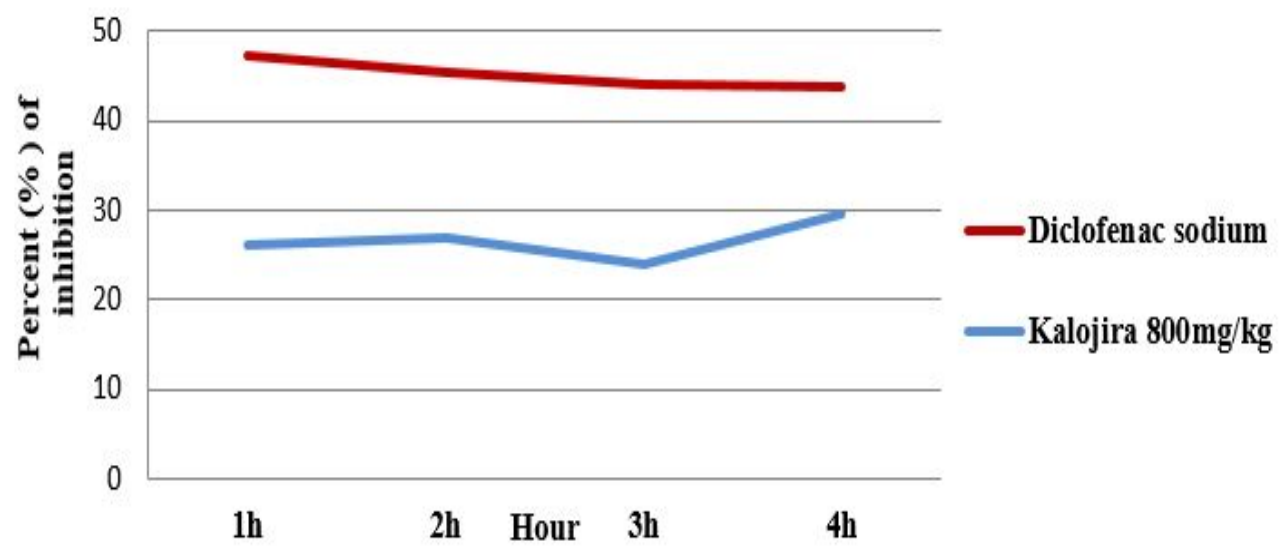

Fig.2. Time course in the changes of percent inhibition on carrageenan-induced paw edema volume of $\mathrm{BC}$ at 1, 2, 3, and $4 \mathrm{~h}$ at the dose of $800 \mathrm{mg} / \mathrm{kg}$, p.o. 


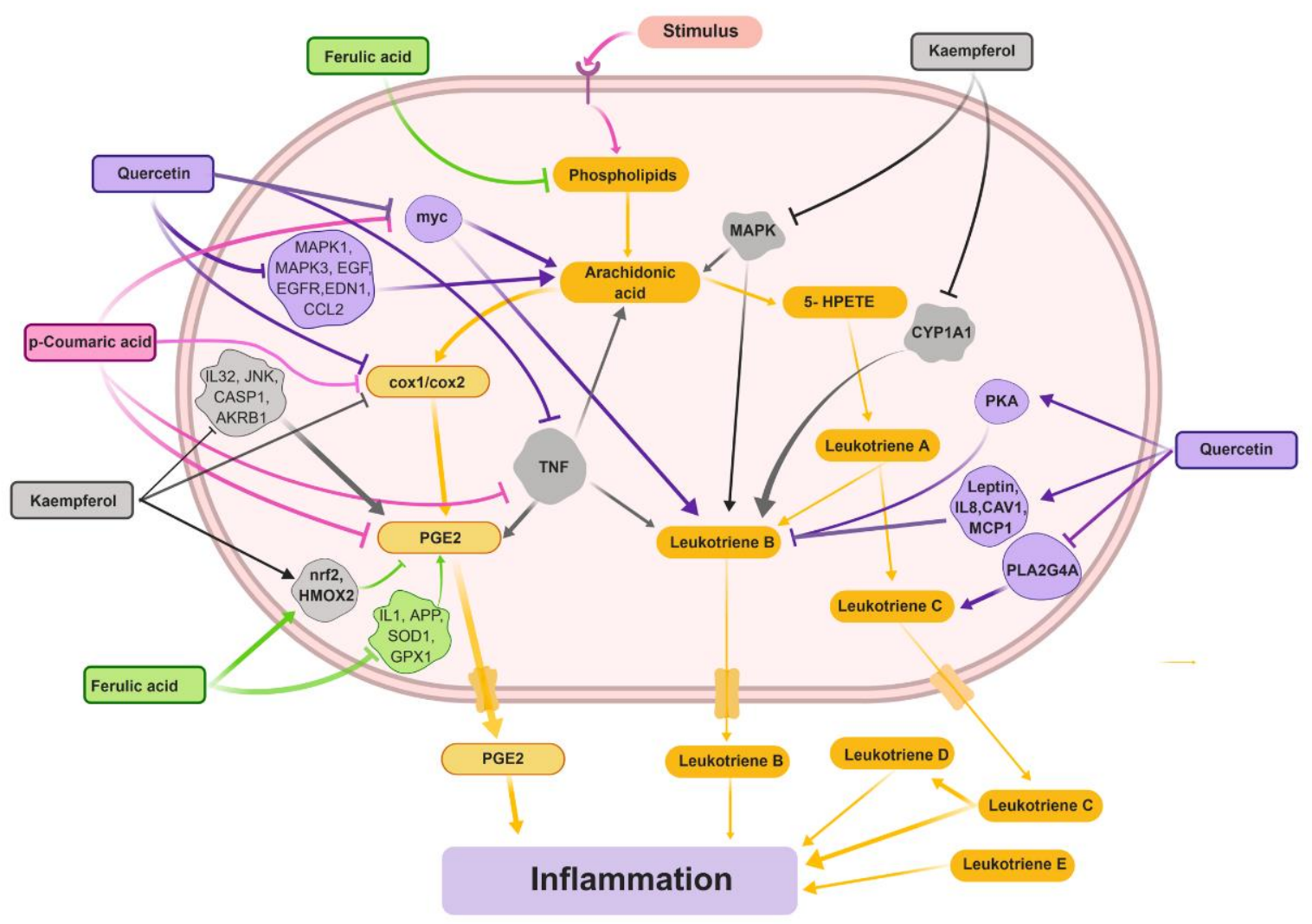

Fig. 3. Possible mechanism of action of BC extracts to reduce inflammation in humans. Phenols and polyphenols, e.g., ferulic acid, p-coumaric acid, kaempferol, and quercetin in the BC extracts, inhibit the signaling molecules, stimulation of which may directly or indirectly result in inflammation. Proteins linked to inflammation participate in several canonical pathways underlying a range of biological activities. Ingenuity pathway analysis (IPA) identified that the BC extracts' components could target many of these proteins (Table S1) and control inflammation. Arrows $(\downarrow)$ indicate direct stimulatory modification, whereas light up signs $(T)$ indicate an inhibitory modification. The Names of the proteins followed the Hugo nomenclature, NCBI Entrez, UniProtKB/Swiss-Prot system. 
659 Table 1: Effects Black Cumin extracts by DMSO extracts of $B C$ on carrageenan-induced 660 paw in rats

\begin{tabular}{|c|c|c|c|c|c|}
\hline \multirow[t]{2}{*}{ Group } & \multirow{2}{*}{$\begin{array}{l}\text { Dose } \\
(\mathrm{mg} / \mathrm{kg}, \\
\text { p.o) }\end{array}$} & \multicolumn{2}{|c|}{$\begin{array}{l}\text { Paw } \\
(\mathrm{ml})(\% \text { of inhibition) }\end{array}$} & \multicolumn{2}{|c|}{ volume } \\
\hline & & $1 \mathrm{hr}$ & $2 \mathrm{hr}$ & $3 \mathbf{h r}$ & $4 \mathrm{hr}$ \\
\hline Control & -- & $0.83 \pm 0.02$ & $0.96 \pm 0.02$ & $1.07 \pm 0.02$ & $1.18 \pm 0.03$ \\
\hline \multirow[t]{2}{*}{ Diclofenac sodium } & 50 & $\begin{array}{l}0.44 \pm 0.0^{*} \\
47.18\end{array}$ & $\begin{array}{l}0.53 \pm 0.02^{*} \\
45.32\end{array}$ & $\begin{array}{l}0.60 \pm 0.01^{*} \\
44.10\end{array}$ & $\begin{array}{l}0.66 \pm 0.01^{*} \\
43.79\end{array}$ \\
\hline & 200 & $\begin{array}{l}0.71 \pm 0.01^{*} \\
14.15\end{array}$ & $\begin{array}{l}0.78 \pm 0.01^{*} \\
19.03\end{array}$ & $\begin{array}{l}0.89 \pm 0.02^{*} \\
16.26\end{array}$ & $\begin{array}{l}0.94 \pm 0.03^{*} \\
20.62\end{array}$ \\
\hline \multirow[t]{2}{*}{ Nigella sativa } & 400 & $\begin{array}{l}0.63 \\
0.001^{*} \\
23.39\end{array}$ & $\begin{array}{l}0.74 \pm 0.01^{*} \\
23.52\end{array}$ & $\begin{array}{l}0.83 \pm 0.001^{*} \\
21.88\end{array}$ & $\begin{array}{l}0.85 \pm 0.001^{*} \\
27.69\end{array}$ \\
\hline & 800 & $\begin{array}{l}0.61 \pm 0.01^{*} \\
26.21\end{array}$ & $\begin{array}{l}0.70 \pm 0.01^{*} \\
26.99\end{array}$ & $\begin{array}{l}0.81 \pm 0.01^{*} \\
24.06\end{array}$ & $\begin{array}{l}0.83 \pm 0.01^{*} \\
29.66\end{array}$ \\
\hline
\end{tabular}

${ }^{\text {a }}$ presented as mean $\pm \operatorname{SEM}(\mathrm{n}=3)$

* $p<0.05$ compared with the control group (Dunnett's test)

661

662

663 Table 2: Effect of Black Cumin extracts by DMSO on acetic acid-induced (0.6\%) writhing 664 in mice

\begin{tabular}{llll}
\hline Group & $\begin{array}{l}\text { Dose } \\
(\mathbf{m g} / \mathbf{k g},(\mathbf{p . o} .)\end{array}$ & No. of writhing ${ }^{\text {a }}$ & \% inhibition \\
\hline Control & -- & $23.67 \pm 1.2$ & -- \\
Diclofenac sodium & 50 & $14.70 \pm .89^{* *}$ & 37.89 \\
& 200 & $18.01 \pm 1.0^{*}$ & 23.91 \\
Nigella sativa & 400 & $17.35 \pm 0.67^{*}$ & 26.65 \\
& 800 & $16.62 \pm 0.33^{*}$ & 29.78 \\
\hline
\end{tabular}

${ }^{\mathrm{a}}$ presented as mean $\pm \operatorname{SEM}(\mathrm{n}=3)$

* $\mathrm{p}<0.05$ compared with the control group (Dunnett's test)

${ }^{* *} \mathrm{p}<0.001$ compared with the control group (Dunnett's test)

665 